\title{
Transatlantica
}

Revue d'études américaines. American Studies Journal

1 | 2017

Morphing Bodies: Strategies of Embodiment in Contemporary US Cultural Practices

\section{L'identité américaine et le détour par l'étranger : Permanence et réinvention du récit de voyage aux États-Unis}

11 \& 12 janvier 2018, Université Grenoble Alpes - Site Marguerite

Soubeyran (Valence)

Marie Thévenon

(2) OpenEdition

Journals

Édition électronique

URL : https://journals.openedition.org/transatlantica/8829

DOI : $10.4000 /$ transatlantica.8829

ISSN : $1765-2766$

Éditeur

Association française d'Etudes Américaines (AFEA)

Référence électronique

Marie Thévenon, «L'identité américaine et le détour par l'étranger : Permanence et réinvention du récit de voyage aux États-Unis », Transatlantica [En ligne], 1 | 2017, mis en ligne le 29 novembre 2018, consulté le 21 mai 2021. URL : http://journals.openedition.org/transatlantica/8829 ; DOI : https:// doi.org/10.4000/transatlantica.8829

Ce document a été généré automatiquement le 21 mai 2021

\section{cc) (†) $\ominus$}

Transatlantica - Revue d'études américaines est mise à disposition selon les termes de la licence Creative Commons Attribution - Pas d'Utilisation Commerciale - Pas de Modification 4.0 International. 


\section{L'identité américaine et le détour par l'étranger : Permanence et réinvention du récit de voyage aux États-Unis}

11 \& 12 janvier 2018, Université Grenoble Alpes - Site Marguerite Soubeyran (Valence)

\section{Marie Thévenon}

1 Conçu comme une collaboration entre spécialistes de civilisation et de littérature, ce colloque s'inscrit dans le projet quinquennal de l'axe «études américaines» du CEMRA/ILCEA4 (Université Grenoble Alpes), intitulé « From Utopia to Heterotopia and Beyond ». Il s'agit d'explorer en quoi le voyage et le mouvement ont un rôle structurant dans l'identité américaine et d'analyser leur impact et leur influence sur les pratiques universitaires et la pensée. Le mouvement et le voyage jouent en effet un rôle important dans la constitution du champ des études américaines et le but de ce colloque est de revenir sur le mouvement dans le temps long, d'explorer le «Travel Writing ", terme plus générique, plus ouvert que le récit de voyage. C'est toute cette Amérique et sa pensée en mouvement qu'il faut saisir, cette dialectique de la distance et de la familiarité, de l'étrange et de l'autochtone entre les espaces et les périodes.

2 La journée d'étude du 17 novembre 2016 s'était intéressée au "mouvement dans la culture américaine ». Le prochain colloque, fin 2019, sera consacré aux « non-lieux » et à la description des États-Unis comme lieu de la négation (anti-espaces, anti-Europe...). L'étude portera également sur la figure de l'étranger avec ses implications politiques en cette période de repli nationaliste.

Lors du colloque des 11 et 12 janvier, qui rassemblait chercheurs confirmés et débutants, le thème commun des communications était celui de l'exploration de l'identité américaine, soit à partir du témoignage d'étrangers, soit de l'intérieur, en commençant par la période de la fin du XVIII ${ }^{\mathrm{e}}$ siècle et du début du XIX ${ }^{\mathrm{e}}$ dans une approche chronologique. Ainsi, Hortense de Villeneuve, la plus jeune chercheuse 
invitée, a débuté ce colloque avec une analyse du récit de voyage des Michaux, père et fils, deux botanistes français, et l'émergence d'une mythologie liée à une république commerciale. La problématique de la filiation est au cœur de cette communication et le corpus étudié met en avant le fait que l'intérêt (terme dont la polysémie est particulièrement intéressante) pour le nouveau monde change à cette époque. Dans son analyse du récit des Innocents Abroad, Frédéric Dumas, quant à lui, explore l'émergence de l'archétype du touriste à une époque marquée par une oisiveté improductive gouvernée par la logique de la consommation ostentatoire. Frédéric Dumas met en avant l'ambivalence de ce récit qui met en scène l'ignorance (des touristes mais aussi du narrateur) comme le meilleur moyen de préserver une innocence américaine menacée par l'élitisme et la prétention. Par bien des aspects, le récit de voyage de Carnegie qu'expose ensuite Carole Masseys-Bertonèche représente le pendant de celui de Twain. En effet, dans les textes de Carnegie, c'est toute la mythologie du voyage américain qui est réactivée pour contribuer à légitimer le capitalisme brutal des " captains of industry » ainsi qu'à conférer une patine de respectabilité culturelle à leur entreprise mercantile. La mise en récit proposée par Carnegie permet de rendre compte des transferts de modèles transatlantiques qui façonneront de manière décisive l'Amérique de la fin du $\mathrm{XIX}^{\mathrm{e}}$ et du début du $\mathrm{XX}^{\mathrm{e}}$ siècle. Audrey Bonvin, quant à elle, revient sur les chroniques de la voyageuse suisse Adèle Huguenin dans les États-Unis de 1913. Militante féministe et anti-alcoolique, Huguenin se retrouve confrontée à une société urbaine, vulgaire, commerciale et anarchique où les réalités locales échappent à un ordre fédéral. Pierre-Antoine Pellegrin s'intéresse au mythe brisé du grand voyageur chez Kerouac. Aux États-Unis, où tout est trop cartographié, il n'est plus possible de connaître l'aventure. Kerouac va donc chercher à l'étranger ce qui a disparu aux États-Unis. L'hétérotopie mexicaine se présente à lui comme le négatif même des États-Unis, l'autre côté du miroir, ou, comme dirait Foucault, un de ces lieux absolument différents, un de ces « contre-espaces ». Mais la rencontre avec l'ailleurs semble impossible. Pour Dominique Mantelli, Eddy L. Harris semble offrir un cas d'école pour penser cette difficulté qu'ont les Afro-Américains à se faire une place dans une Amérique qui les rejette et, par conséquent, à se confronter à leur statut de communauté diasporique. Pour Harris, cette condition est décrite à travers l'écriture de voyage qui permet de superposer le récit de soi et le récit de voyage dans un effort pour constituer ce qu'il appelle une "auto-topographie». Clément Rodier s'intéresse à Adorno qui dépeint les États-Unis comme un endroit où la pensée disparaît, où elle est considérée seulement dans sa dimension d'objet pratique. La situation fluctuante d'Adorno (il est dedans et il est aussi en-dehors) fait de lui un observateur idéal, mais le place dans une situation intenable. Clément Rodier tient cependant à nuancer cette image d'un intellectuel isolé et explique qu'il existe une certaine porosité entre Adorno et les États-Unis. Bertrand Naivin travaille sur la photographie et choisit de montrer une série de photographies illustrant «l'étranger » en rappelant que la photographie américaine procède d'une approche différente de l'européenne avec un ancrage dans une représentation du monde qui se veut très efficace: il s'agit de faire naître et d'entretenir la culture du présent. Enfin, Isabelle Sinic-Bouhaouala montre comment les manuels d'histoire du secondaire aux États-Unis tentent de réorganiser le récit national autour de la figure du voyage et de l'étranger. En effet, depuis les années 90, les manuels d'histoire tentent de s'éloigner du récit exceptionnaliste de l'histoire américaine et de l'émergence historique d'une nation caractérisée par ses institutions 
politiques en insistant résolument sur l'émergence d'une communauté d'expérience autour de la figure de l'étranger/immigré.

Bon nombre de ces communications problématisent le rapport de l'altérité et de l'identité, du proche et du distant, de la domesticité et de l'exil (cf. Kerouac, Adorno, Harris). De même, si la critique des topos associés au récit de voyage procède en partie d'un désenchantement à l'égard de l'Amérique prosaïque/matérialiste des $\mathrm{XIX}^{\mathrm{e}}$ et $\mathrm{XX}^{\mathrm{e}}$ siècles, elle peut aussi participer d'un refus de renoncer à l'idéal du voyage qui doit être radicalement redéfini (Twain et Kerouac). Enfin, les communications insistent également sur la difficulté de rendre compte d'une nation qui serait fondamentalement caractérisée par le mouvement.

\section{Jeudi 11 janvier}

\section{Hortense de Villeneuve (Université Paris-Sorbonne) : « La naissance d'une nation vue par les Michaux : 'a commercial republic' »}

Hortense de Villeneuve propose d'analyser le récit de voyage des Michaux, père et fils, tous deux botanistes français, en se focalisant particulièrement sur le voyage de François-André Michaux effectué de 1801 à 1803 et rapporté dans Voyage à l'Ouest des Monts Alleghany et dans les États de l'Ohio, Kentucky et Tennessee. Elle présente d'abord André Michaux, le père (1746-1802), qui est un botaniste naturaliste, autodidacte, polyglotte et grand voyageur. Envoyé en Amérique par Louis XVI pour enrichir les pépinières royales en raison d'une pénurie de bois en France, il profite de l'occasion pour s'insérer dans les réseaux d'intellectuels américains. Son fils, François-André Michaux, lui succédera et deviendra responsable des pépinières, qu'il devra fermer pour des raisons financières. Il voyage lui aussi beaucoup et fait preuve d'une grande curiosité scientifique en prenant beaucoup de notes sur la flore qu'il observe avec passion et ses écrits assouviront la curiosité du grand public. La problématique centrale de la filiation attire notre attention sur l'idée de transition, de passage de génération: de la figure du père qui incarne l'impersonnalité typologique du botaniste des lumières (voire une figure du colon qui recense les ressources du nouveau monde à exploiter) à la posture plus ambiguë du fils qui, s'il propose un récit qui s'éloigne résolument des conventions du récit d'aventure pittoresque (du fait qu'il est motivé par des considérations mercantiles), tend avant tout à définir les contours d'un modèle social dont le narrateur se sent tout à la fois proche et distant. L'hésitation générique et pragmatique du récit ainsi que l'ambiguïté de la posture narrative adoptée font ainsi écho au fait que le récit de voyage cherche à se redéfinir dans une société américaine qui est elle-même en transition à cette époque et interrogent l'idée de nation définie par Marcel Mauss.

\section{Frédéric Dumas, Université Grenoble Alpes : « Mark Twain d'Amérique en Amérique : la visite guidée des innocents »}

6 Le point de vue est ici celui d'un Américain écrivant sur son propre pays en rassemblant les observations qu'il a faites au cours de ses voyages. C'est le premier de ceux-ci, la croisière du «Quaker City » en 1867 narrée dans The Innocents Abroad (1869), qu'analyse Frédéric Dumas. Il s'agit du récit d'un voyage d'agrément qui évoque le 
déclin du voyageur et l'avènement du touriste, le voyage de ce dernier étant voué à la seule recherche du plaisir. Mark Twain décrit des touristes ethnocentriques et ignorants dont les loisirs représentent une forme de consommation tout à fait représentative de l'esprit de cette époque. Il dénonce les pulsions consuméristes de touristes qui n'hésitent pas à voler et à vandaliser les lieux explorés. Twain joue le rôle du satiriste critiquant une Amérique du Gilded Age qui a pris ses distances par rapport à l'éthos originel du Nouveau Monde. L'émergence de l'archétype du touriste est donc métonymique de cet âge dans la mesure où il est marqué par une oisiveté improductive gouvernée par la logique de la consommation ostentatoire théorisée par T. Veblen. Cette logique du déclin est reflétée dans la structure du récit qui renverse de manière parodique la logique du Grand Tour (via notamment l'assimilation comique du touriste à la figure du Barbare et du Philistin) mais aussi par la logique de l'accélération, de la répétition et de la prétérition qui ne donne rien à voir ainsi que par l'insistance sur le motif de la vanité thématisé tant par les champs de ruines visités que par les pratiques de ces touristes. Pourtant, Frédéric Dumas insiste aussi sur l'ambivalence de ce récit qui met en scène l'ignorance (des touristes mais aussi du narrateur) comme le meilleur moyen de préserver une innocence américaine menacée par l'élitisme et la prétention (et on peut voir l'humour comme une forme d'écriture démocratique). Indirectement, la critique du monumental et la mise en avant du vagabondage constituent une critique de la quête des origines, des racines, ce qui confère d'autant plus de poids à la découverte épiphanique d'Ephèse qui représente une réactivation de l'expérience américaine de l'identification du voyageur à l'espace qui échappe en partie au langage et à la logique du récit. En somme, si Twain critique la mythologie dégradée du voyage promue par le tourisme, il parvient à la réactiver. C'est l'américanité des voyageurs qui est mise à l'épreuve dans le voyage.

\section{Carole Masseys Bertonèche, Université de Bordeaux : « L'influence de l'exil et des voyages sur la pensée de Carnegie : du darwinisme anglais au capitalisme philantropique américain »}

7 Carole Masseys Bertonèche propose d'examiner comment Andrew Carnegie, l'un des symboles du "Gilded Age » et l'archétype même du self-made man, a, par sa pensée et par le rôle structurant qu'a joué sur lui le voyage, contribué à l'évolution des institutions américaines. La façon dont Carnegie a souhaité mettre en scène sa propre histoire d'immigré écossais visait en effet à consolider le mythe du "self-made man » mais aussi à se servir de la structure du récit de voyage pour formaliser l'idée des ÉtatsUnis comme une terre de progrès. Ceci est particulièrement clair dans la division de son récit en deux parties : le voyage aller qui reprend largement les codes du récit d'aventure (émerveillement, danger, durée...) alors que le retour s'étonne des transformations qui ont pu avoir lieu en si peu de temps (de l'Ecosse de son enfance, des États-Unis et de Carnegie lui-même). Le récit d'Andrew Carnegie reflète le contraste entre son identité d'immigré et son statut social élevé d'homme américain. La fable de la réussite personnelle est un décalque de la marche inéluctable du progrès incarnée par les États-Unis et se reflète dans le fait que le retour dans le vieux monde ne peut être que décevant. Il s'agit d'un récit fortement marqué par un idéal aristocratique et élitiste qui a eu tendance à minimiser la radicalité politique de sa famille ou à revenir sur la dimension problématique de la révolution industrielle. De même, la mise en avant de l'idéal américain de liberté (lieu commun associé au récit de voyage dans le 
nouveau monde) est justement utilisée pour justifier un capitalisme débridé assimilé, par le truchement de Darwin et Spencer, à une expression contemporaine d'un état de nature édénique où «all is well since all is getting better ». Toutefois, ce que Carole Masseys Bertonèche illustre bien à travers ce cas, c'est justement que si Carnegie parvient par le truchement de ce récit à s'investir des attributs de l'homme américain, il parvient aussi à acclimater dans le nouveau monde des théories (Darwin et Spencer justement) et des outils juridiques (notamment celui du trust qui date du XIV siècle) venus du Royaume-Uni. Comme Oxbridge, les universités américaines sont des « feeder schools »: les parents envoient leurs enfants là où ils ont eux-mêmes étudié. Les universités ont les moyens de rechercher les étudiants dont le profil les intéresse et de prendre qui elles veulent. Il s'agit en réalité, pour elles, d'un retour sur investissement. Carnegie va d'ailleurs écrire en 1886 son essai Triumphant Democracy pour défendre l'idée que la démocratie américaine est l'accomplissement de la monarchie libérale britannique.

Les questions portent d'abord sur l'étymologie du terme " philanthropie ». Ce concept est-il d'origine américaine ou non ? Le terme est alors comparé au mécénat, ce modèle "aristocratique" qui intervient surtout dans le domaine des arts, alors que la philanthropie englobe des champs multiples. On ne peut comprendre les États-Unis si on ne comprend pas le fonctionnement et l'importance du système philanthropique.

\section{Vendredi 12 janvier}

\section{Audrey Bonvin, Université de Fribourg, Suisse : «'Je n'ai vu aucune de ces merveilles' : chroniques de la voyageuse suisse Adèle Huguenin dans les États-Unis de 1913 ou quand La Petite Lumière se fait sur les (re)présentations de la prohibition »}

En se basant sur son carnet de voyage, ses chroniques journalistiques, ses lettres personnelles adressées à la "Womens' Christian Temperance Union » et à une amie, ainsi que sur des coupures de presse d'époque, Audrey Bonvin décrit le voyage d'Adèle Huguenin, militante féministe et antialcoolique. Auteur de romans populaires qui racontent des histoires heureuses, empreintes de morale, elle est membre du parti socialiste et de l'Union des femmes pour le bien, s'intéresse aux problèmes sociaux en Suisse et s'en prend au bouc émissaire de l'époque, l'alcool, et tout particulièrement l'absinthe, dont la production a remplacé l'activité des ateliers d'horlogerie et de dentellerie régionale. Du fait de son appartenance aux mouvements socialistes, féministes et antialcooliques, elle participe au développement des réseaux transnationaux qui se structurent au début $\mathrm{du} \mathrm{XX}^{\mathrm{e}}$ siècle. C'est sa correspondance avec Marthe Maret qui donne naissance à l'idée de partir en tournée aux États-Unis. Partie de Liverpool, Adèle Huguenin parvient à Philadelphie en 1912, et poursuit son voyage en passant par Washington, New York, le New Jersey et le Connecticut, pour arriver enfin à Boston. Ce voyage est pourtant très décevant car elle ne peut que constater à quel point le gouvernement américain est hypocrite: nul n'ignore que les gens, s'ils s'abstiennent pendant la semaine, boivent le dimanche. Elle se rend compte que les méthodes suisses de lutte contre l'alcool sont bien supérieures à celles que l'on pratique aux États-Unis. Ce voyage sera le seul qu'elle effectuera car elle en sort épuisée. Il s'agit donc là d'une rencontre qui n'a pas abouti et qui arrive presque à contretemps. En effet, 
comme en atteste sa correspondance, ce voyage ne permet pas à Adèle Huguenin de confirmer la parenté qu'elle croit deviner entre la démocratie américaine et la confédération suisse (renforcée par le poids de la religion et un imaginaire rural) qui permettrait justement de faire avancer la cause antialcoolique. Ce récit pragmatique, marqué par la frustration, fait contre-point au récit hyperbolique et sensationnaliste offert par la presse américaine.

\section{Pierre Antoine Pellerin, Université Lyon 3 : « L'hétérotopie mexicaine dans les récits de Kerouac : entre fantasme orientaliste et tourisme sexuel »}

10 Pierre Antoine Pellerin commence sa communication en insistant sur le statut paradoxal de cette icône américaine dont, comme on le sait, l'anglais n'était pas la langue maternelle. Il s'agit d'une figure de l'exil qui ne se reconnaîtra ni dans sa Bretagne natale (évoquée dans Satori in Paris) ni dans les États-Unis dont il considère qu'ils ont été dénaturés par la prospérité des années cinquante. Cette critique s'exprime notamment à travers divers essais écrits pour Holiday Magazine où Kerouac considère que le double processus de modernisation et de domestication de l'espace américain a contribué à priver le voyageur de la découverte de l'inconnu et de la jouissance qui l'accompagne. C'est ainsi que l'œuvre de cet auteur est constamment tendue entre le rejet du voyage assimilé à la vacuité de l'activité touristique et le désir de fuir une mère-patrie féminisée incarnant tous les maux d'une vie castratrice faite de déceptions et de malheurs. Kerouac semble trouver la solution à ce dilemme dans l'idéalisation de la maison close mexicaine qui représente ainsi un lieu affranchi des lois du foyer mais représente aussi un espace hétérotopique qui existe en dehors, en marge de la réalité et où une nouvelle communion fraternelle et primitive peut être mise en scène. Pierre Antoine Pellerin insiste sur l'idéalisation de ces descriptions ainsi que sur le sous-texte colonial de cette mise en scène qui puise abondamment dans l'imaginaire d'une Afrique étrangère et primitive. Mais, en mettant en contraste les récits de Kerouac avec les Mexican Memoirs de Bonnie Bremser, il montre aussi combien ces voyages représentent une forme d'appropriation non seulement des corps des prostituées mais aussi des espaces qui ne sont en définitive que la surface sur laquelle se projette une identité collective et personnelle d'une Amérique fantasmée.

11 Une discussion s'engage sur le rôle de la femme dans le voyage, sur ces femmes qui sont souvent considérées comme un obstacle chez Kerouac, le voyage étant un concept très masculin. La femme n'est envisagée que dans le rôle de mère ou de prostituée dans ce discours de la crise de la masculinité face à la féminisation de la société. Est également posée la question du désenchantement : les protagonistes de Kerouac ne cherchent-ils pas le danger, la menace, le risque de perdre la vie, ce qui va à l'encontre de l'idéal pionnier ? Chez Kerouac, il y a en effet une mise en scène de soi, une projection de ce qu'on croit ne pas pouvoir trouver aux États-Unis et qu'on ne peut découvrir que par le voyage. C'est ainsi qu'on constate une certaine fascination pour l'Afro-Américain et ce désir d'altérité dans On the Road, qu'on retrouve aussi chez Miller, ainsi que chez Burroughs. 


\section{Dominique Mantelli, Université de Rouen : «Le récit de voyage comme zone de traduction : la place de 'l'étranger' dans l'auto- topographie de l'écrivain noir américain Eddy L. Harris »}

Dominique Mantelli commence par une brève évocation de la biographie et de la bibliographie de Eddy L. Harris, en insistant sur le rapport de l'auteur avec sa couleur de peau. Harris se construit à travers le regard de ceux qui l'entourent, majoritairement des blancs aisés : "I'm not African, I am a product of the culture that raised me " (Mississipi Solo,13) ; " I am an American. And I am Black " (Native Stranger, 28). Il voue un amour particulier à la France (le français, appris à l'école, est sa deuxième langue), et il va s'efforcer, au fil de ses voyages, d'acquérir un véritable bilinguisme. Lorsqu'il évoque ses écrits, il réfute le terme de "récit de voyage ", lui préférant l'expression "travel narratives ». Dominique Mantelli évoque le rôle de la France dans la construction de ses récits par le prisme de la traduction. Eddy L. Harris s'efforce de mettre en évidence la difficulté qu'ont les Afro-Américains à se faire une place dans une Amérique qui les rejette et, par conséquent, à se confronter à leur statut de communauté diasporique. Cette condition d'exil est bien entendu le fruit de la double conscience théorisée par W.E.B. Du Bois (qui réduit l'identité à une altérité imposée par le détour d'un regard blanc qui lui est étranger), à quoi s'ajoute le fait que le père de Harris, qui passait pour blanc, ne lui a pas davantage offert la possibilité de s'ancrer dans un récit, une genèse afro-américaine. Pour Harris, cette condition est décrite à travers l'écriture de voyage qui permet de superposer le récit de soi et le récit de voyage dans un effort pour constituer ce qu'il appelle une " auto-topographie ». Le Mississippi fonctionne comme un topos majeur de cette écriture (viz. Mississippi Solo) tout comme la France qui, paradoxalement, représente non seulement un lieu où le narrateur peut échapper à sa condition raciale devenue invisible mais aussi s'approcher autant que faire se peut d'une Afrique qui demeure à jamais inaccessible. La liberté existe dans cet entre-deux. Ce qui est d'autant plus intéressant dans le cas de Harris, c'est la façon dont ces problématiques sont amplifiées par le travail et le rôle joué par la traduction dans son écriture ainsi que par une pratique éditoriale complexe qui contribue à brouiller davantage encore l'idée d'une origine, d'une langue maternelle, d'un texte source.

\section{Clément Rodier, Université de Bordeaux : «Theodor W. Adorno et les 'United Stat's ”}

13 Le cas d'Adorno évoqué par Clément Rodier représente un cas limite pour cette journée d'étude dans la mesure où le philosophe de l'école de Francfort exilé aux États-Unis pendant la guerre n'a pas produit de récit de voyage à proprement parler même si l'Amérique constitue un lieu crucial de son écriture et y introduit une forme d'étrangeté. En effet, pendant les années trente, Adorno définit les États-Unis, son positivisme utilitariste, sa recherche planifiée et son fétichisme des faits, comme l'altérité contre laquelle se constitue une théorie critique qui considèrerait la pensée comme une force émancipatrice capable de rendre compte des circuits de domination. En cela, les États-Unis sont un avatar non seulement d'une modernité technocapitaliste contre laquelle la théorie critique se devait de lutter mais aussi le berceau de cette philosophie analytique qui gagnait de plus en plus d'influence en Europe grâce au 
cercle de Vienne. Cette posture rhétorique va perdurer lors des années d'exil aux ÉtatsUnis voire même être renforcée par l'échec du Princeton Radio Project avec Paul Lazarsfeld. Elle s'exprime non seulement dans les lettres d'Adorno à Horkheimer ou ses études sur le jazz mais aussi dans les Minima Moralia que Clément Rodier considère comme le produit de cette situation d'exil: le caractère fragmentaire de l'ouvrage reflète non seulement la violence de cet arrachement de la pensée adornienne à son contexte mais exprime également son refus d'assimiler les impératifs fonctionnels et techno-scienfiques de son pays d'accueil en tentant de préserver un espace de réflexion éminemment personnel. Cependant, Clément Rodier insiste bien sur l'artificialité de la posture insulaire adoptée par l'intellectuel allemand et invite à nuancer cette posture d'observateur critique. Si la théorie critique adornienne a progressivement su trouver toute sa place dans le paysage de la recherche américaine dans la seconde moitié du $\mathrm{XX}^{\mathrm{e}}$ siècle, Adorno, comme il a été dit, a également choisi de prolonger ses collaborations transatlantiques après être rentré en Allemagne et a continué de revenir aux États-Unis tout au long de sa vie. Par ailleurs, des œuvres tardives, comme La Personnalité autoritaire, rendent compte d'une inflexion méthodologique notable dans la mesure où elles ménagent une place importante à la réflexion collective et pluridisciplinaire, à l'observation empirique et aux données quantitatives.

Les questions qui suivent la communication s'attachent surtout au parallèle qu'on pourrait dresser entre Adorno et Tocqueville. Est également discuté le rapport d'Adorno à la langue anglaise : certains de ses textes ont été écrits en anglais, mais il tenait beaucoup à préserver la langue allemande. Ses écrits personnels, rappelle-t-on, étaient rédigés en allemand.

\section{Bertrand Naivin, Université Paris VIII : «L'identité américaine ou le détour par la photographie de l'étranger »}

Bertrand Naivin rappelle qu'il y a eu deux naissances de la photographie : l'une est européenne, l'autre est américaine. Cette dernière procède d'une approche différente avec un ancrage dans une représentation du monde qui se veut très efficace. Sa contemporaine européenne, bien différente, s'inscrit dans la tradition de la grande peinture. Le poids créé par le respect de cette tradition n'existe pas aux États-Unis où il s'agit de faire naître et d'entretenir la culture du présent, comme le montre l'exemple du Pop Art avec ses objets neufs et son présent éternel. Bertrand Naivin présente alors une série de photographies montrant " l'étranger »: Maxime Ducamp, Otto Schoefft (représentation de l'Egypte) ; Alphonse Delaunay, Charles Nègre (rapport de la photographie à la peinture) ; Peter La Cherre (cf. Chef Indien dans un style proche du naturalisme) ; John Carbutt (présent en train de se faire) ; William H. Jackson, Edward Curtis (culture du « en train de ») ; Jacob Riis (les États-Unis comme terre d'accueil avec Ellis Island) ; Lewis Hine (scènes domestiques) ; Arnold Genthe (images prises sur le vif) ; Alfred Stieglitz (naissance de la «straight photography »). Naivin évoque pour finir le "selfie» et ce retour de l'immédiat, une forme d'expression contemporaine très importante caractérisée par une véritable obsession de se voir et de partager dans un monde sans époque, en pleine déconstruction où «le drame est un jeu ». Ainsi, cette obsession pour le présent des photographes américains les incite à inventer de nouveaux procédés techniques (l'appareil Kodak, l'objectif grand angle et le selfie) qui permettent non seulement à la photographie de se démocratiser et de mettre en avant 
une esthétique de l'instantané mais aussi de faire de la photographie un art de la mobilité.

Un débat s'engage autour de l'importance du titre donné aux photographies : est-ce le photographe qui donne le titre, et, dans ce cas, est-ce que cela en fait un écrivain ? Détail important, en ce qui concerne les selfies, le texte est souvent inscrit sur la photo puisqu'il s'agit d'une image faite pour communiquer par sms ou par email. Bertrand Naivin revient enfin sur l'invention de l'« instant photography » qui permet de voir la photo dès sa prise (très appréciable dans le cadre familial). Il s'agit d'abord d'une naissance technique avant la naissance de l'art photographique.

\section{Isabelle Sinic-Bouhaouala, Université Grenoble Alpes: " History as a story well told : mise en récit de l'expérience migratoire dans les manuels d'histoire américaine aux États-Unis »}

17 L'identité américaine est en perpétuelle construction et l'étranger y joue un rôle important avec une mise en contraste des opposés. L'identité américaine commence en effet par le voyage, celui des colons anglais. Mais c'est à partir de la rupture avec la Grande-Bretagne que naît réellement l'Histoire américaine. La transmission de l'identité américaine se fait par les manuels d'histoire. Mais comment y définit-on les États-Unis ? Quelle place y réserve-t-on aux " native Americans » et aux afroaméricains ? Peut-on réellement parler « d'étrangers » dans une telle nation? Isabelle Sinic-Bouhaouala explore ainsi la redéfinition de l'immigrant, ou comment "l'autre » devient le «même » dans les manuels scolaires qui font de l'expérience migratoire le socle fondateur, avec une vision aussi bien spatiale qu'historique, où le regard de l'autre joue le rôle de processus de construction de l'identité américaine. Isabelle SinicBouhaouala montre ainsi comment les manuels d'histoire récents dans l'enseignement secondaire aux États-Unis, tels que America Will Be (Houghton Mifflin, 1993), The Americans (Houghton Mifflin, 2012), America Past and Present (Pearson, 2013), Discovering our Past: A History of the United States (McGraw Hill Education, 2017) et History Alive! The United States Through Modern Times (Teachers' Curriculum Institute, 2014), tentent de réorganiser le récit national autour de la figure du voyage et de l'étranger. En mettant en avant la nature organique d'une identité commune qui est constamment en devenir, ces manuels répondent bien entendu à la diversité grandissante du public scolaire et à la montée des préoccupations collectives autour des questions d'identité mais aussi à un désir de prendre ses distances avec le programme social associé à des récits dont la vocation est résolument assimilationniste, construits à la fin du XIX et au début du XX siècle. Non seulement ces manuels insistent sur un temps très long qui permet de marquer une continuité d'expérience entre Native Americans, colons, afro-américains, migrants du $\mathrm{XIX}^{\mathrm{e}}$ et du $\mathrm{XX}^{\mathrm{e}}$ siècles, mais ils insistent aussi sur la dynamique de la rencontre (par opposition à la découverte ou la conquête) et s'appuient sur un vaste corpus de récits de voyages personnels - réels ou fictionnels - qui permettent de problématiser l'opposition binaire entre étranger et autochtone. En somme, plutôt que de chercher à établir un récit unique ou à définir ce que seraient les traits de la nation, ces manuels utilisent le récit de voyage et la figure de l'étranger pour décrire le devenir commun d'une communauté nationale caractérisée par une pluralité de trajectoires, de récits et d'expériences. 
INDEX

Thèmes : Actualité de la recherche

\section{AUTEUR}

MARIE THÉVENON

Université Grenoble Alpes 